\title{
Ultrafast Reaction Pathways in a Metalloprotein Revealed by Optical Polarization Selected X-ray Transient Absorption Spectroscopy and Quantum Mechanical Calculations
}

\author{
M. L. Shelby, ${ }^{1}$ D. Hayes, ${ }^{2}$ P. Lestrange, ${ }^{3}$ K. Fransted, ${ }^{2}$ H. Lemke, D. Zhu, X. Li, L. X. Chen,,${ }^{1,2}$ \\ ${ }^{1}$ Department of Chemistry, Northwestern University, Evanston, IL 60208; \\ ${ }^{2}$ Chemical Sciences and Engineering Division, Argonne National Laboratory, Lemont, IL 60439; \\ ${ }^{3}$ Department of Chemistry, University of Washington, Seattle, WA 98195; \\ ${ }^{4}$ LCLS, SLAC National Laboratory, Menlo Park, California 94025, USA
}

Excited state pathways and electronic structural dynamics during photodissociated CO from heme in myoglobin is investigated by ultrafast X-ray transient absorption (XTA) spectroscopy using femtosecond laser pump and X-ray probe approach at the Linac Coherent Light Source (LCLS), an X-ray free electron source and by quantum mechanical calculations. The optical polarization selected OPS-XTA revealed dynamics, analogous to optical anisotropy, of $\mathrm{Fe}-\mathrm{C}(\mathrm{O})$ bond breakage and accompanying electronic spin state transition as well as nuclear geometry evolution with the relative polarization of the laser and X-ray beams parallel and perpendicular with each other. We have demonstrated that a polarization dependent dichroism of the evolution of the XANES signal can be detected and interpreted even for an excited population with relatively low order compared to systems usually studied by polarization-dependent XAS, which represents an additional dimension of structural and electronic information that can be extracted from ultrafast XAS. We have interpreted the polarization dependence of global fit kinetic fit components towards a mechanism for myoglobin active site relaxation. In sum, the data indicates a rapid displacement of iron out of the heme plane which occurs with a time constant of $80 \mathrm{fs}$ as $\mathrm{CO}$ dissociates, accompanied by a low spin to high spin transition and the compression of the Fe-NHis bond. Movement of the F-helix, elongation of the Fe-NHis bond, and expansion of the Fe-Np bonds occur simultaneously with a time constant of $0.89 \mathrm{ps,} \mathrm{while} \mathrm{the} \mathrm{initial} \mathrm{relaxation} \mathrm{of} \mathrm{in-plane} \mathrm{modes}$ occurs with a time constant of $3.4 \mathrm{ps}$. The $\mathrm{CO}$ departure pathway is mapped out by a set of quantum mechanical calculations using Fe-CO distance and the ring doming as main reaction coordinates which assigned the pre-edge feature evolution on the femtosecond time scale and it correlation with the spin state transition. 